\title{
Silicon Photomultipliers for the LHCb Upgrade Scintillating Fibre Tracker
}

\author{
Guido Haefeli* and Zhirui $\mathrm{Xu}^{\dagger}$ \\ EPFL Lausanne, Switzerland \\ E-mail: guido.haefeli@epfl.ch
}

\begin{abstract}
Silicon photomultipliers (SiPMs) are solid state photodetectors that combine all of the features necessary for the photon detection of a high resolution Scintillating Fibre Tracker (SciFi Tracker). Two SiPM manufacturers, Hamamatsu and KETEK have developed customized devices for SciFi Tracker application in the context of the LHCb tracker upgrade. These custom devices provide high photon detection efficiency (PDE) in a large wavelength range, high reliability due to its simple mechanical construction, a high density multi-channel package and are of sufficiently low cost to build a large area tracking device. There are several challenging requirements placed on the photodetector mainly due to the neutron radiation environment and the low light output of the long scintillating fibre modules. New devices with the latest technological improvements implemented are available in spring 2014. This includes devices with different optical isolation between pixels (trenches) and different pixel sizes. The dark noise rate (DCR) increases strongly with irradiation and the noise cluster rate of the tracking device can only be kept sufficiently low at a temperature of $-40^{\circ} \mathrm{C}$. We present the results on PDE, cross-talk and noise before and after neutron irradiation at various temperatures. The results for the new devices are compared to the devices based on the standard technology.
\end{abstract}

Technology and Instrumentation in Particle Physics 2014,

26 June, 2014

Amsterdam, the Netherlands

\footnotetext{
* Speaker.

${ }^{\dagger}$ On behalf of the LHCb SciFi Tracker group
} 


\section{Introduction}

The LHCb detector [1] in its initial configuration, will collect data until the second long shutdown (LS2) of the LHC accelerator. The detector granularity, the readout, and the trigger scheme are limiting the current detector performance. A major detector upgrade [2] scheduled during LS2 from mid 2018 to the end of 2019, will extend the physics reach of the experiment by allowing higher luminosity and higher trigger efficiency. To achieve this, the upgrade relies on two major changes. Firstly, the readout at full interaction rate, currently limited by a Level-0 trigger rate of $1 \mathrm{MHz}$, will be introduced (no hardware trigger). Secondly, the upgraded LHCb detector will be designed to cope with an increased luminosity by an order of magnitude by improving the detector granularity and its data acquisition system.

In the current detector, the forward tracking region is divided into an inner tracker (IT) region (12 layers, total surface $3 \mathrm{~m}^{2}$, pitch $200 \mu \mathrm{m}$, silicon strip detectors) and an outer tracker (OT) region (12 layers, total surface $300 \mathrm{~m}^{2}, 2.5 \mathrm{~m}$ long, $5 \mathrm{~mm}$ drift tubes). To fulfill the requirements for the upgrade, the fine granularity of the inner region has to be extended. The main requirements on the upgraded tracking detector are:

- Hit detection efficiency larger than $98 \%$ with noise cluster rate less than $10 \%$ of the signal cluster rate

- Spatial resolution for single hits better than $100 \mu \mathrm{m}$

- $40 \mathrm{MHz}$ readout

- High occupancy, up to 2.5 clusters for a detector array of 128 channels $(32 \mathrm{~mm})$ in the hottest region

- Low material budget, smaller than $X / X_{0} \leq 1 \%$ per detection layer

- Radiation environment at SiPMs after integrated luminosity of $50 \mathrm{fb}^{-1}$ is $6 \times 10^{11} n_{\text {eqv }} / \mathrm{cm}^{2}$ with neutron shield (the neutron shield reduces at least a factor of two). Ionising radiation at the center of the detector is $35 \mathrm{kGy}$ (for fibers) and at the location of the SiPM $100 \mathrm{~Gy}$.

The LHCb collaboration has chosen to cover the full IT and OT region with a fine pitch scintillating fiber tracker. In view of the SciFi Tracker [3] construction, studies to optimize the detector for its application were performed and are ongoing within the LHCb SciFi group. Hereafter we will focus on the optimization and performance of the photodetectors, silicon photomultipliers (SiPMs) regarding all important aspects of the SciFi Tracker application. This includes neutron irradiation studies for devices from different manufacturers as Hamamatsu ${ }^{1}$ and KETEK ${ }^{2}$.

\section{Signal description and mechanical aspects}

The signal generated by a particle and its detection in the SiPM array is illustrated in Fig. 1 (left), taken from the output of a Geant 4 simulation. A particle is crossing almost perpendicular to the fibre mat and generates light in several fibres. The light is transported directly or via mirror to the detector. Black dots show the position of the photons arrive on the detector after propagation in fibres. These photons arriving on the detector can trigger an avalanche in a pixel (fired pixels in yellow) and the probability is the photon detection efficiency (PDE) of the detector. The channels

\footnotetext{
${ }^{1}$ Hamamatsu Photonics K.K., 325-6, Sunayama-cho, Naka-ku, Hamamatsu City, Shizuoka Pref., 430-8587, Japan.

${ }^{2}$ KETEK GmbH, Hofer Str. 3, 81737 München, Germany.
} 

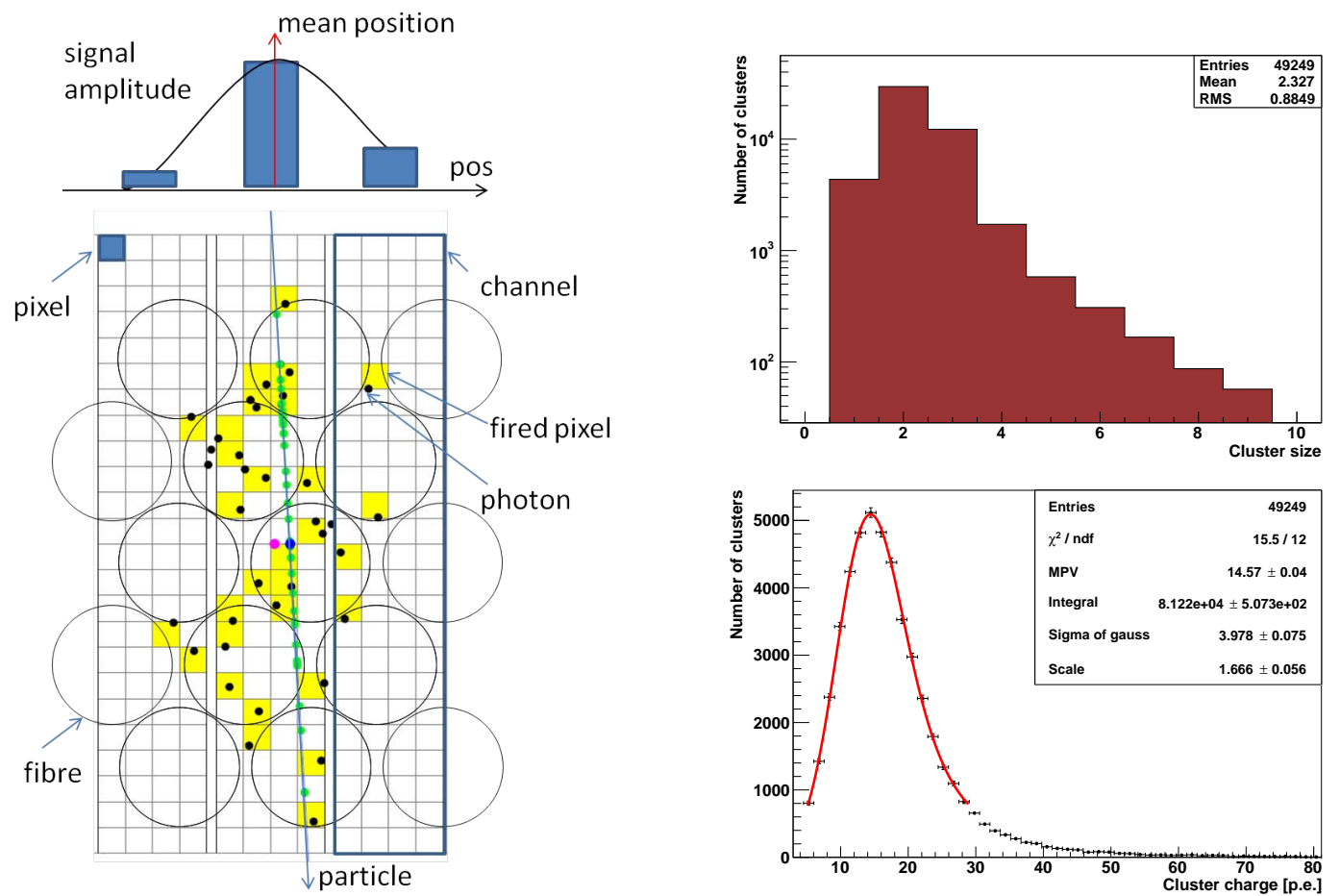

Figure 1: Left: Illustration of a Geant4 generated event. The photons produced along the trajectory of the particle are propagated to the fibre end and further to the detector. Each pixel of the detector can detect one photon and the signal proportional to the total number of fired pixels (coloured pixels) is the signal amplitude per channel illustrated in the top part of the figure. The particle position can be calculated with a weighted mean value of the channel signal. Note that the fibre pitch is $275 \mu \mathrm{m}$ while the SiPM channel width is $250 \mu \mathrm{m}$. Right: Signal characteristics from a fibre module, (top) cluster size (bottom) cluster signal distribution.

of the SiPM array are outlined and consist of $4 \times 24$ pixels. The channel dimension is adapted to the fibre diameter and the number of fibre layers. Optimisation studies showed that with fibres of $250 \mu \mathrm{m}$ the channel size of $250 \mu \mathrm{m}$ is best suited. Smaller channels will not improve the spatial resolution since the photons are randomly distributed within fibres and larger channel size will worsen the resolution. The channel height for five layers of fibers is $1.32 \mathrm{~mm}$ and for six layers is $1.5 \mathrm{~mm}$ for Hamamatsu devices and $1.68 \mathrm{~mm}$ for KETEK devices. The channel height allows for at least $100 \mu \mathrm{m}$ alignment tolerances between fibre and SiPM. The glue between the fibres is loaded with $\mathrm{TiO}_{2}{ }^{3}$, a white powder, to reduce optical cross-talk (x-talk) between fibres and therefore improve spatial resolution. The width difference between SiPM channel and fibre pitch introduces a random alignment between fibre and detector channel which has no negative consequences for the spatial resolution.

Each pixel produces the same amount of signal given by the gain of the detector. The total signal in a channel is the sum of all pixels fired. Digital processing is used to combine the information from several channels to a cluster, based on several threholds. The position of a hit is given as the

\footnotetext{
${ }^{3}$ For the 2.5 meters long fibre modules of $\mathrm{LHCb}$, the light output of the module is the most critical parameter. We are optimizing the $\mathrm{TiO}_{2}$ concentration in the glue to obtain the best possible signal (optical cross-talk and signal amplitude).
} 
weighted mean of the cluster.

The signal can be characterised by distribution of cluster size and the distribution of total number of photons detected per cluster, which are shown in Fig. 1 (right). The signal from a perpendicular crossing particle can be spread over one, two or three channels (for geometrical reasons) but larger spreads due to x-talk between fibres, between SiPM pixels and delta electrons in the signal production, lead to a cluster size distribution as given in Fig. 1 (top right) (measurement with cosmic rays). Note that the cluster size distribution is strongly dependent on the clustering algorithm, its thresholds setting and the light recorded for a minimum ionising particle (MIP) value. The MIP value is the most likely value of the number of photons per cluster measured. With the same clustering algorithm, the cluster size distribution is wider for a larger MIP value.

The silicon of the detector and the fibre end are separated with a thin transparent protection layer: epoxy is used for Hamamatsu devices and glass plate for KETEK devices. The protection layer is used to protect the bonding wires on the surface and the silicon from oxidation. The thickness of the layer is desired to be as thin as possible because a photon emitted by the fibre is displaced from the fibre exit point according to its exit angle and the thickness of the layer. With the simulation and the experimental setup the thickness of 100-120 $\mu \mathrm{m}$ was found to be acceptable without degradation of the spatial resolution and no significant increase of the cluster size.

\section{The noise cluster rate}

The noise cluster rate depends on the thresholds of the clustering algorithm, the dark count rate (DCR, Fig. 2), the x-talk and after-pulsing and the electronic shaping. The DCR increases with over-voltage and temperature, the total surface of the detector and the neutron fluence. To measure the performance of the detector many of these parameters can be fixed because they are imposed by system requirements.

- The operation temperature is fixed to $-40^{\circ} \mathrm{C}$. Whereas lower temperatures would impact heavily on the cooling design (dry environment and isolation).

- The over-voltage is fixed to the range where PDE is not increased significantly anymore. Maximised PDE with lowest possible DCR is the aim. A typical operation point is at $3.5 \mathrm{~V}$ over-voltage.

- The electronics shaping has an upper limit fixed by the LHC bunch spacing of $25 \mathrm{~ns}$ and a lower limit given by the sum of signal dispersion due to the large detector size ( $2.5 \mathrm{~m}$ long) and the scintillator decay time of the SCSF-78MJ fibre of $2.8 \mathrm{~ns}$. The signal propagation time in the fibre is $15 \mathrm{~ns}$ for $2.5 \mathrm{~m}$ (direct signal) and up to $30 \mathrm{~ns}$ for mirrored signal. Strong attenuation of the mirrored signal, due to the long path, reduces the latest arriving light arriving light.

- The size of the channel is given by the fibre mat thickness and the channel width and the total area per channel is $0.33 \mathrm{~mm}^{2}$ for five layers and $20 \%$ larger for six layer detectors.

- The radiation environment simulation allows to estimate the neutron fluence to be $6 \times 10^{11} n_{\text {eqv }} / \mathrm{cm}^{2}$ with a neutron shield or $12 \times 10^{11} n_{\text {eqv }} / \mathrm{cm}^{2}$ without neutron shield. It is not clear at the time if the shield can be installed everywhere in the detector.

The noise suppression is processed on the front-end (FE) card near the SiPM detectors in order to reduce the data bandwidth. The large DCR of the SiPM can efficiently be suppressed using 
the threshold-based clustering algorithm. This algorithm has proven its performance to distinguish signal from noise and allows to reduce the DCR of $10 \mathrm{MHz}$ per channel to a noise cluster rate below $5 \mathrm{MHz}$ per 128 channel array. New detector structures to reduce DCR after irradiation and reduced $x$-talk (after-pulse with low influence) remain as possible improvements. In Fig. 2, the noise cluster rate is plotted as a function of different $\mathrm{x}$-talk values. We observe a large increase of the noise cluster rate due to $\mathrm{x}$-talk which motivates low $\mathrm{x}$-talk value.
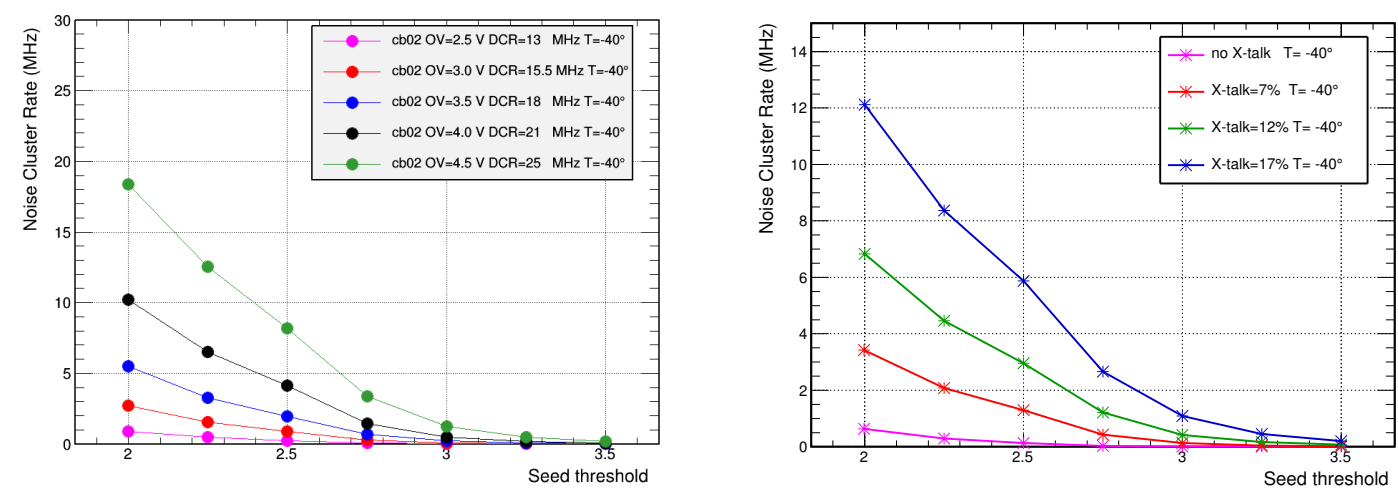

Figure 2: Noise cluster rate as a function of the thresholds in the clustering algorithm. Left: Simulation of the noise cluster rate for different over-voltages (DCR and $\mathrm{x}$-talk dependence on over-voltage taken into account). Right: Noise cluster rate for different $\mathrm{x}$-talk probabilities. Simulated plots for $0.33 \mathrm{~mm}^{2}$ area and a neutron fluence of $6 \times 10^{11} n_{\text {eqv }} / \mathrm{cm}^{2}$ (after anneling).

\section{Summary of the results obtained from Hamamatsu and KETEK detectors}

To evaluate the performance of the detectors we measure gain, $\mathrm{x}$-talk, temperature dependence of breakdown voltage and the PDE as a function of wavelength before irradiation. Irradiation was performed at an installation at EPFL based on several Pu-Be sources allowing irradiation at room temperature with a rate of $2 \times 10^{10} n_{\text {eqv }} / \mathrm{cm}^{2}$ per day. In this setup, annealing is taking place during the irradiation. Additional annealing yielding $40 \%$ DCR reduction after one week at $40{ }^{\circ} \mathrm{C}$ was typically observed. After irradiation the DCR of the detectors was measured at cold temperature which in our case is -20 to $-60^{\circ} \mathrm{C}$. For these measurements, the bias voltage was adjusted for the operation temperature according to the results from non-irradiated characterisation. No measurable effect for the gain, $\mathrm{x}$-talk and PDE was observed due to radiation.

The high level of DCR at the end of life time of the experiment requires particularly low $\mathrm{x}$-talk in order to keep the noise cluster rate sufficiently low. The detectors from the generation without trenches were modified with optical barriers to avoid pixel to pixel x-talk. Single channel devices from Hamamatsu and multichannel devices from KETEK are compared with and without trenches as shown in Fig. 3. At an over-voltage of $3.5 \mathrm{~V}$ corresponding to a gain of $2 \times 10^{6} \mathrm{e} / \mathrm{PE}$, the Hamamatsu single channel detectors have a $\mathrm{x}$-talk of $7 \%$ with a very small after-pulse contribution. KETEK has added in a technology evaluation production an additional trench (double trench) which efficiently reduces the $\mathrm{x}$-talk to below $2 \%$ at an over-voltage of $3.5 \mathrm{~V}$ at a gain of 
$9.5 \times 10^{6} e / P E$. With the slow shaping where the after-pulse contributes to the $\mathrm{x}$-talk value, it is increased to $3 \%$. Note that the $\mathrm{x}$-talk is expected to be proportional to the charge released by an avalanche (gain) for a given technology.
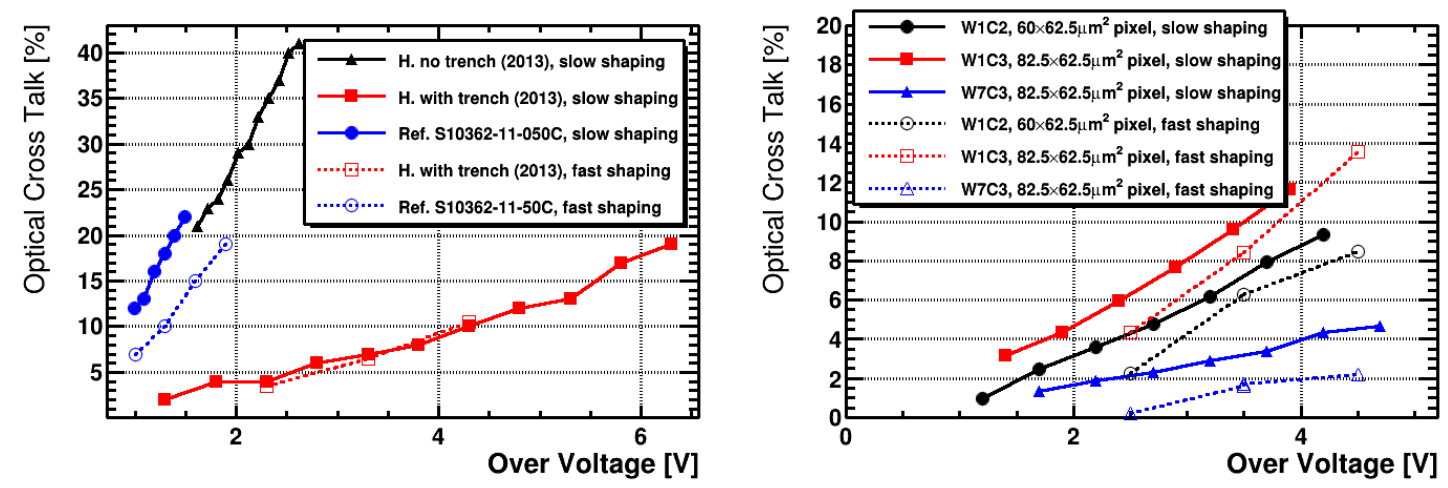

Figure 3: Comparison of $x$-talk as a function of over-voltage for different SiPM options. Left: Hamamatsu single channel devices; Right: KETEK multichannel devices (W1C2 and W1C3 with trenches and W7C3 with double trenches). The dotted lines correspond to the $\mathrm{x}$-talk measurements with a very fast shaping and allows therefore to exclude the contribution from after-pulse. The difference between dotted and full lines is the contribution by after-pulsing.

The DCR increase due to irradiation and its decrease by lowering the operation temperature is quantified by measuring the I-V curve at different temperatures (Fig. 4). The plots are using a relative scale for the bias voltage (over-voltage) in order to provide an easy comparison between different detectors at different temperatures. To achieve high PDE the devices has to be operated at high over-voltage with the cost of a higher DCR. The KETEK devices show a similar DCR at $-40^{\circ} \mathrm{C}$ as the Hamamatsu devices. For different technologies a factor 4 difference of the DCR values are observed.
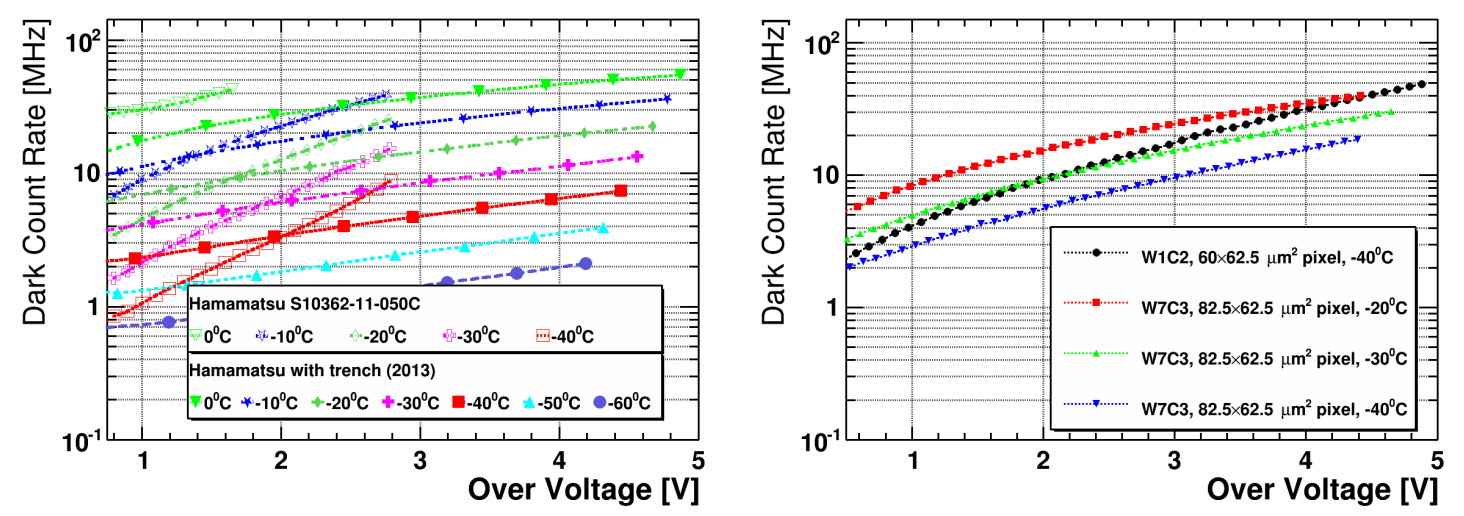

Figure 4: DCR as a function of over-voltage at different temperatures. Left: Hamamatsu devices; Right: KETEK devices. Note that the DCRs were scaled to $0.33 \mathrm{~mm}^{2}$ area and a neutron fluence of $2 \times 10^{11} n_{\text {eqv }} / \mathrm{cm}^{2}$ (after anneling) was applied. 
The temperature dependence of the DCR is altered with radiation and also changes with different SiPM technologies. The reduction of DCR by a factor 2 is obtained if the detector is cooled by a temperature difference $K_{1 / 2}$. The exponential dependence is seen linear in the logarithmic plots (Fig. 5). For irradiated Hamamatsu devices, $K_{1 / 2}$ is about $10 \mathrm{~K}$ without trench and $12.8 \mathrm{~K}$ with trench and 15-18 K for KETEK devices with trenches. The different slopes in Fig. 5 (right) also indicate the changes of the $K_{1 / 2}$ value before and after irradiation.
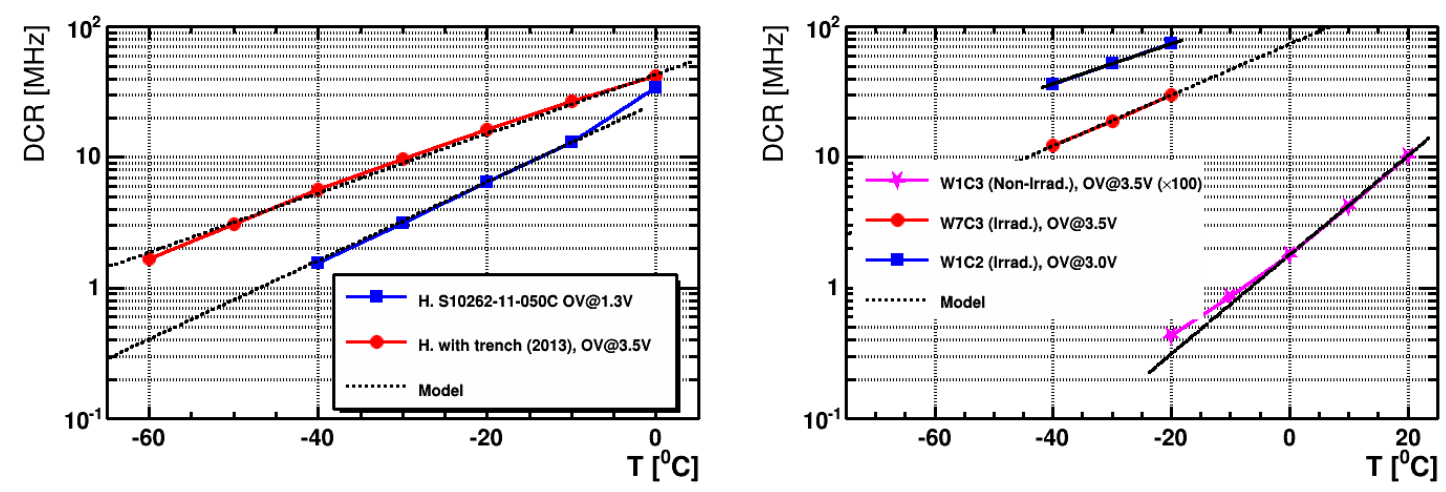

Figure 5: Dark count rate dependence as a function of temperature at a certain working over-voltage for different SiPMs. Left: Irradiated Hamamatsu devices $\left(2 \times 10^{11} n_{\text {eqv }} / \mathrm{cm}^{2}\right)$; Right: Irradiated KETEK devices $\left(2 \times 10^{11} n_{\text {eqv }} / \mathrm{cm}^{2}\right)$, and for comparison, a non-irradiated KETEK device (a scale factor of 100 multiplied).

For our PDE measurement, we use the Hamamatsu multichannel array $50 \mu \mathrm{m}$ pixel detectors (device based on the same technology as the S10262-11-050C) as the reference. The PDEs plotted are all $\mathrm{x}$-talk, after-pulse and dark current corrected. The Hamamatsu reference technology has a PDE of $30 \%$ at $1.3 \mathrm{~V}$ over-voltage. The wavelength dependent PDE of the Hamamatsu with trench and a KETEK device at different over-voltages are given in Fig. 6. To increase the PDE the detectors with trenches are operated at higher over-voltage, typically $3.5 \mathrm{~V}$ for our application. Note that the emission spectrum of an irradiated long fibre is green shifted compared to the SiPM sensitivity. A green shifted technology would result in more light for the detector, which is partially obtained by increasing the over-voltage.

\section{Summary}

For LHCb SciFi Tracker we evaluated custom SiPM arrays from KETEK and Hamamatsu, which both have high PDE, low x-talk and sufficient radiation hardness. The KETEK technology shows a very high peak PDE where for the Hamamatsu the broad sensitivity gives an advantage for the green shifted emission spectrum of the fibre. Detectors with (double) trenches have shown extremely low x-talk and are good candidates for the SciFi Tracker application. The high DCR can be reduced by cooling - an operation temperature of $-40^{\circ} \mathrm{C}$ reduces the noise to an acceptable level. The temperature dependence of the DCR is dependent on the SiPM technologies and is different before and after irradiation. 

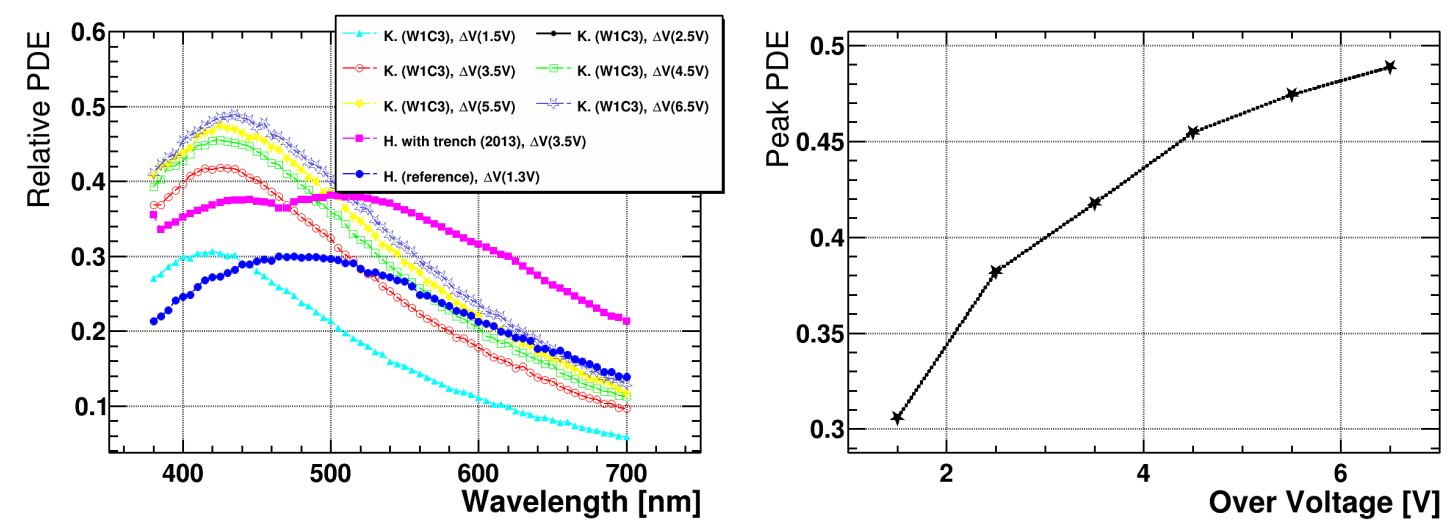

Figure 6: Left: Photon detection efficiency as a function of wavelength measurement for different SiPMs. Right: The peak PDE as a function of over-voltage for the KETEK W1C3 detector. Note that all plots are $\mathrm{x}$-talk corrected.

\section{References}

[1] LHCb Collaboration, A. A. Alves Jr. et al., The LHCb detector at the LHC, JINST 3 (2008) S08005.

[2] LHCb Collaboration, R. Aaij et al., Framework TDR for the LHCb Upgrade, CERN-LHCC-2012-007, LHCb TDR 12.

[3] LHCb Collaboration, LHCb Tracker Upgrade Technical Design Report, LHCB-TDR-015, Feb. 2014. 